\title{
Clinical Trial for Assessing the Effectiveness and Tolerability of the Medical Device Sunekos 200, Applied with Electroporator in Skin Ageing
}

\author{
Patrizia Sacchi ${ }^{1}$ and Raffaele Rauso ${ }^{2 *}$ \\ ${ }^{1}$ Aesthetic physician in private practice, Italy \\ 2Professor Maxillo-Facial Surgery, University of Campania “Luigi Vanvitelli”, Italy
}

*Corresponding author: Prof Raffaele Rauso, Maxillo-Facial Unit, University of Campania, "Luigi Vanvitelli", Piazza Miraglia, 80138-Napoli, Italy, Tel: 00393397772305; Email: dr.raffaele.rauso@gmail.com

\section{Research Article \\ Volume 3 Issue 2}

Received Date: May 08, 2018

Published Date: June 14, 2018

DOI: $10.23880 /$ cdoaj-16000152

\section{Abstract}

Background: Assessment of the effectiveness and tolerability, with regard to facial photoageing, of a solution containing a blend of amino acids and low molecular weight hyaluronic acid (Sunekos 200), delivered using an electroporator, capable of stimulating the production of new collagen and elastin.

Target: The objective of this study was to verify whether the same effects, in terms of effectiveness and tolerability, are achieved when delivering the product Sunekos 200 with an electroporator rather than injecting it.

Method: The study was performed on healthy female patients, aged between 45 and 65 years old. 30 patients were treated on the face and 10 patients were treated on the hands. All patients underwent 4 sessions of electric traction every week. The patients were assessed at baseline, after 4 weeks and 1 month after the end of the sessions (T0-T1-T2), using validated clinical scales, subjective and objective assessments (skin check-up) and a photographic evaluation (T0-T1-T2). All the patients were asked to answer a self-assessment questionnaire after the end of the treatment.

Results: In all cases, the results obtained showed an improvement in all the clinical and subjective assessments and in most of the objective instrumental parameters. In any case, the clearest results appear in the space of approximately one month, with a distinct visible improvement in the degree of ageing of the skin, its hydration and sebum levels. The overall evaluation expressed by both the volunteers and the testers (Oesse Total Checkup - Casale Monferrato (AL) - Italy), in terms of effectiveness and tolerability, was good/excellent.

Conclusions: The results obtained confirm that by delivering Sunekos 200 with an electroporator, as in the intradermal therapy technique, we obtain an improvement in the hydration of the skin, its sebum levels, firmness, and the number of micro-wrinkles on the surface. The results are less visible and less long-lasting than those achieved by introducing the device with intradermal therapy technique. 
Delivering the product by way of electroporation to the hands proved extremely effective, almost immediately generating results. This type of application is in any case preferable for all patients with needle phobia or with a particularly active social life. Based on the objective results and those resulting from the clinical analyses, this type of application method can be a valid alternative to the traditional intradermal treatment method.

Keywords: Sunekos 200; Skin Ageing; Photoageing; Intradermal therapy; Electroporator

Abbreviations: HA: Hyaluronic Acid; ECM: Extracellular Matrix; BMI: Body Mass Index.

\section{Introduction}

As the years pass, our skin undergoes changes on both a cellular and structural level, leading to a gradual, slow transformation of the person's appearance [1]. Skin ageing is a complex phenomenon with an onset during youth: the first visible signs of ageing on the skin appear on the skin only in the fourth decade of life. It is an irreversible process, but one that can be counteracted and slowed down. In the skin ageing process, changes caused by intrinsic factors overlap, also to different extents from person to person. These changes are related to the genetically programmed chronological ageing process and others are due to extrinsic causes, linked to the environment in which we live and our lifestyle, such as exposure of the skin to the ultra violet rays of the sunlight, pollution, smoking and also diet, the main causes of photo-induced skin ageing or photo-ageing [2-4].

As the years go by, the skin, especially facial skin, undergoes different types of alterations, which appear in the form of:

First signs of ageing: micro-wrinkles, slight accentuation of the fine lines, dullness, slightly loose appearance, skin that appears less toned and elastic.

Notable signs of ageing: deep wrinkles, dry, thin skin, relaxed/saggy appearance due to the "gravitational" effect $[5,6]$.

Then, in women, we must also consider the influence of the hormones on the signs of ageing, as the skin is a hormone-dependent organ and the drop in oestrogens caused by the menopause accelerates the ageing process. This results in a reduction in the sebum levels and the tone of the skin and brings about a number of changes to the epidermis. Indeed, saggy skin is one of the most notable phenomena in the post-menopause period [5].
Anti-ageing treatments can be modulated, based on the requirements of the skin and whether it shows the first signs of ageing, or the signs of ageing are clearer and more visible, without their necessarily being a linear link to the person's age. Additionally, given the importance of hydrating the skin, it is feasible to consider moisturising treatments as the prime cosmetic gesture required to counteract skin ageing $[4,5]$.

The visible signs of ageing depend on a series of changes that involve the various skin layers. As the years pass, the epidermis thins, the cellular renewal process slows down, the production of sebum and the ability of the skin to retain moisture are reduced and the skin therefore becomes drier and more fragile, favouring the formation of wrinkles. Additionally, the pigmentation of the skin can become irregular, and dark spots (the socalled "age spots") appear [4].

Instead, in the dermis, especially due to the effect of the hormonal changes, the production of collagen, elastin and hyaluronic acid is reduced, resulting in the alteration of the connective tissue: the skin thins and loses tone and elasticity, causing the sagginess typical of more mature skins.

At an even deeper level, in the hypodermis, changes linked to the adipose tissue, or subcutaneous fat, take place, with loss of volume and a "sunken" effect especially on the cheeks [7].

The skin on the back of the hands is also affected. It is particularly delicate and constantly exposed to many external agents, from climatic factors to the cleaning and cleansing agents used, it is subject to obvious changes caused by ageing. Indeed, as the years go by, the skin on the hands becomes thinner, losing tone and elasticity. This thinning of the skin makes the veins and tendons annoyingly prominent [6]. 


\section{Clinical Dermatology Open Access Journal}

Various studies have shown that the skin can be stimulated, with intradermal injections of organic substances, capable of inducing the dermis to revitalize itself, and so correct the alterations related to photo and chrono-ageing. In aesthetic medicine, products made with hyaluronic acid (HA) are often used as these are capable of reducing the signs of ageing by improving the tone and elasticity of the skin, due to its natural hydrating and stimulating properties. Hyaluronic acid is also useful for repairing the tissues; it favours the proliferation of fibroblasts, stimulates the synthesis of new collagen and other components of the extracellular matrix (ECM). Additionally, due to its scavenging action on the free radicals, $\mathrm{HA}$ is used to accelerate the healing process in wounds $[7,8]$.

There are different preparations of HA for biorevitalisation with different molecular weights, concentrations and viscosities. The product studied, (Sunekos 200 - professional Dietetics SpA - Milan, Italy) is a medical device containing HA with a low molecular weight and a specific blend of amino acids. Sunekos 200 is capable of promoting the production of new collagen and elastin, through the chemotactic migration of the fibroblasts into the injected area $[9,10]$.

The objective of this work was to verify whether the same effects are achieved when delivering Sunekos with an electroporator rather than injecting it $[8,11]$.

\section{Materials}

\section{Formulations}

Sunekos 200 is a medical device (class III) presented in sterile, apyrogenic vials containing $100 \mathrm{mg}$ of lyophilised amino acids (Glycine, L-proline, L-leucine, L-lysine HCL, Lvaline, L-alanine) and a sterile vial of sodium hyaluronate (30 $\mathrm{mg}$ in $3 \mathrm{ml}$ distilled water) produced and distributed by Professional Dietetics SpA and it is used for correcting the signs of ageing and photo- ageing of the face and body (of light and medium gravity) [12-14].

The product was applied using an Eporex K 69 electroporator (Medical Technology - Rimini, Italy).

All the patients were photographed before treatment, after the end of the sessions and 1 month from the end of the treatment.

All the patients were asked to answer a self-assessment questionnaire after the end of the treatment.
All the patients underwent an instrumental skin checkup before the treatment, after all the sessions and 1 month after the end of the clinical trial. (Oesse Total Checkup - Casale Monferrato (AL) - Italy).

\section{Method}

The transdermal delivery method has been used for a long time. The equipment used in this study is the Eporex K69 system - certified by EC Directive 93/42 EC - fitted with knobs and ionisation chamber.

The equipment uses an innovative method for transporting the molecules, namely iontophoresis. A pulsed, modulated current is used, that is a wave with a low frequency elasto-modulated wave is issued with a modulated intensity that can be regulated by the operator.

The equipment activates the molecules, including those with both low and high molecular weight: this enables them to penetrate deep into the extra-cellular compartment (from 01 to $10 \mathrm{~cm}$ ). In this way, a double effect is achieved: the permeability of the skin tissues is increased, also in the deeper layers of the dermis, and it becomes easier to introduce active hydro-soluble substances with a high molecular weight [12,15-17].

This device has 2 unique characteristics:

1. The modularity of the wave.

2. The presence in the knob of an ionisation chamber.

The presence of the ionisation chamber is essential for the purpose of ensuring the effectiveness of the electroporation: indeed this depends on the type of impulse (duration, width and form) which is applied to the biological tissue and on the type of cell to which it is applied. In the chamber, the blend of active principles is subjected to a fixed and constant induction by the elastomodulated current with different "shapes of wave" typical of the Eporex system.

It is important to underline that the electrical impulse is not released directly on to the skin, but inside the ionisation chamber, where the active principles are located. Once these have been ionised, they pass more easily through the stratum corneum.

The substances penetrate actively thanks to the momentary change of the skin lipids created by the specific electrical impulse and they reach the target cells. 
Another method is also included in the system: "Bright skin". It is based on ultrasound and galvanic currents and serves to intensify the iontophoresis treatment. Bright skin can be used before the application phase, to remove the dead cells from the surface of the skin, so preparing the skin to receive active substances for specific treatments $[12,16,18]$.

\section{Technical Specifications}

\section{EPOREX K69}

- Power voltage: 90V, 220V c.a.

- Working frequency: $50 \mathrm{~Hz}, 60 \mathrm{~Hz}$

- Internal power voltage: $24 \mathrm{Vcc}$

- Maximum absorbed power: $40 \mathrm{VA}$

- Fuses 2x R 2A 250V

- Safety class: I BF

- Maximum output voltage: $45 \mathrm{~V}$

- Maximum output current ( $\mathrm{R}=1 \mathrm{~K}): 45 \mathrm{~mA}$

- Maximum working frequency: $1760 \mathrm{~Hz}$

\section{Brightskin Spatula}

- Power voltage: 24 V c.c.

- Working voltage: 5 V c.c.

- Working frequency: $25 \mathrm{KHz}$

- Max power BRIGHTSKIN SPATULA : $0.1 \mathrm{~mW}$

- Dimensions (LxWxD) 50X20x150 mm

The treatment lasts for 4 weeks, the sessions take place once a week.

In the SUNEKOS 200 protocol it is applied for 30 minutes.

The treatment begins by performing a slight exfoliation with Bright Skin in order to remove the dead cells and facilitate the transfer of the active principles. (in case of different devices a market exfoliation product can be used).

\section{Methodology}

The study was performed on 30 patients of female gender aged between 40 and 65 years old, who were treated on the face and 10 patients of female gender between the ages of 40 and 65 years old who were treated on the hands.

\section{Inclusion criteria}

- Female gender

- Aged between 40 and 65 years old

- Medium/moderate skin photo-ageing according to the photographic scale reference

- Skin phototype I, II, III according to Fitzpatrick's classification

- Absence of exposure to UV rays on the face and hands for the entire duration of the trial.

- Absence of make-up before each treatment

- The patients agreed to maintain: the same diet and sporting habits, the same cosmetics and their usual cleansing products.

\section{Exclusion criteria}

- Pregnancy

- Breast-feeding

- Body mass Index (BMI) variations of more or less 1 during the period of study

- Patients who had previously used permanent fillers in the areas treated

- Patients who had undergone aesthetic treatments (fillers, botulin, bio-stimulations, laser, etc.) in the 6 months prior to the beginning of the treatment.

\section{Assessment of the Effectiveness}

The assessment of the aesthetic results was based on the clinical evaluations, instrumental measurements, a self-assessment questionnaire and 3 pieces of photographic documentation (beginning, end of the treatment and one month after the end of the treatment).

All the assessments were performed in compliance with the standards of environmental conditions, with temperature $22^{\circ} \mathrm{C}+/-2^{\circ} \mathrm{C}$, humidity< $60 \%$ and after a period of at least 15 minutes for the acclimatization of the subject [8].

Primary Endpoint: Objective measurement of the antiageing action based on the instrumental assessment of the changes in comparison with the basal value observed ion the skin after 30 days of treatment, with reference to the following parameters [19-21]:

- Sebum level

- Level of hydration

- $\mathrm{pH}$

- Temperature 
Secondary Endpoints: the clinical assessment was based on the level of photo-ageing according to Glogau, on the condition of ptosis of the face, and the grade of ageing of the nasal and lip folds according to the photographic scale and on the overall anti-wrinkle action: subjective assessment (visual) of the relaxing effect demonstrated on the treated skin, and on the overall performance of the product: self-assessment (questionnaire) given out at the end of the treatment [4-18].

\section{Questionnaire}

\section{Assessment of Tolerability}

The tolerability of the treatment was assessed by taking into account any local events, which were almost completely absent (slight erythema), and any other adverse reactions, also of a systemic origin, which may have occurred during the study [12,13] (Figure 1A-1D).

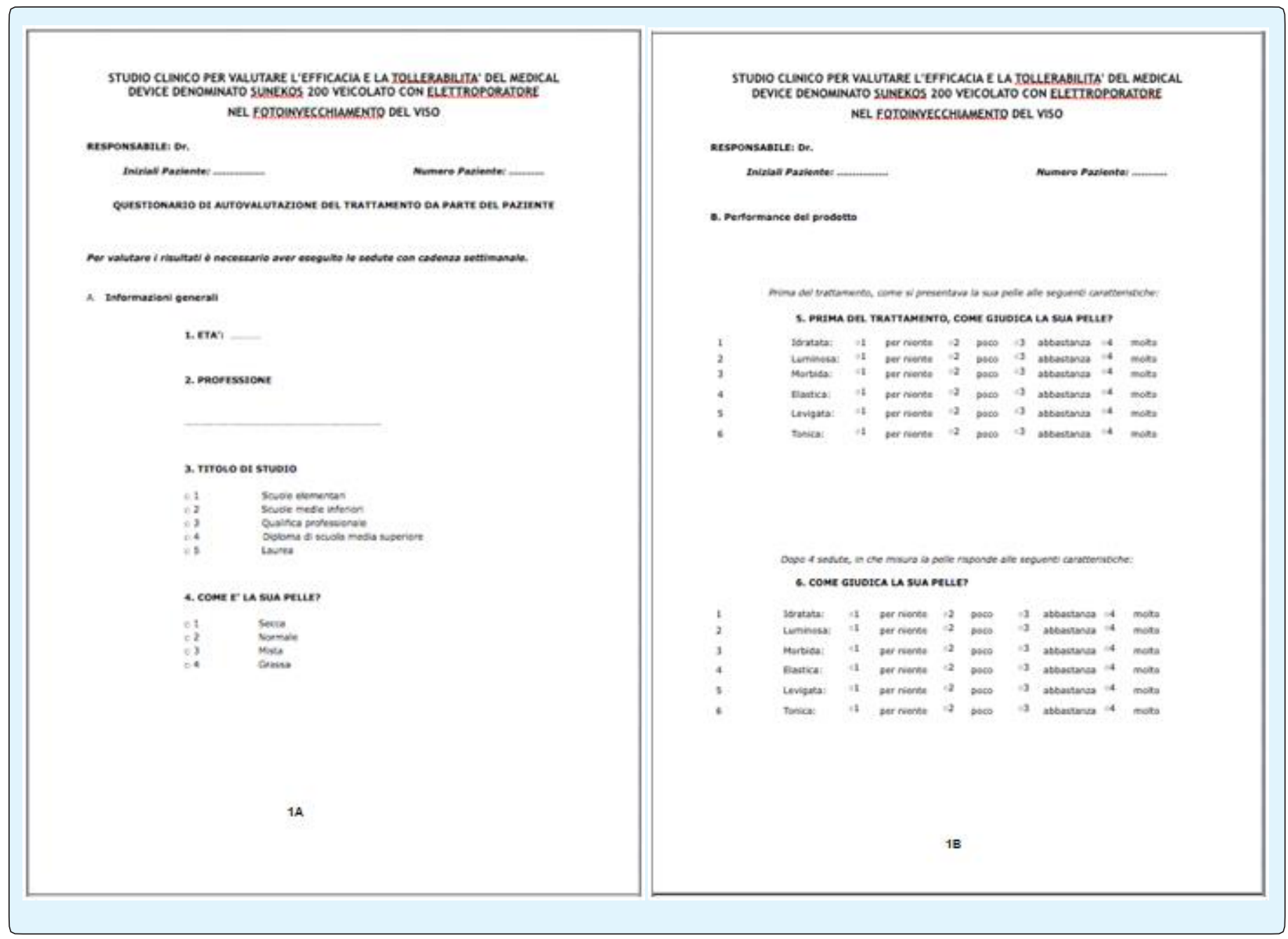




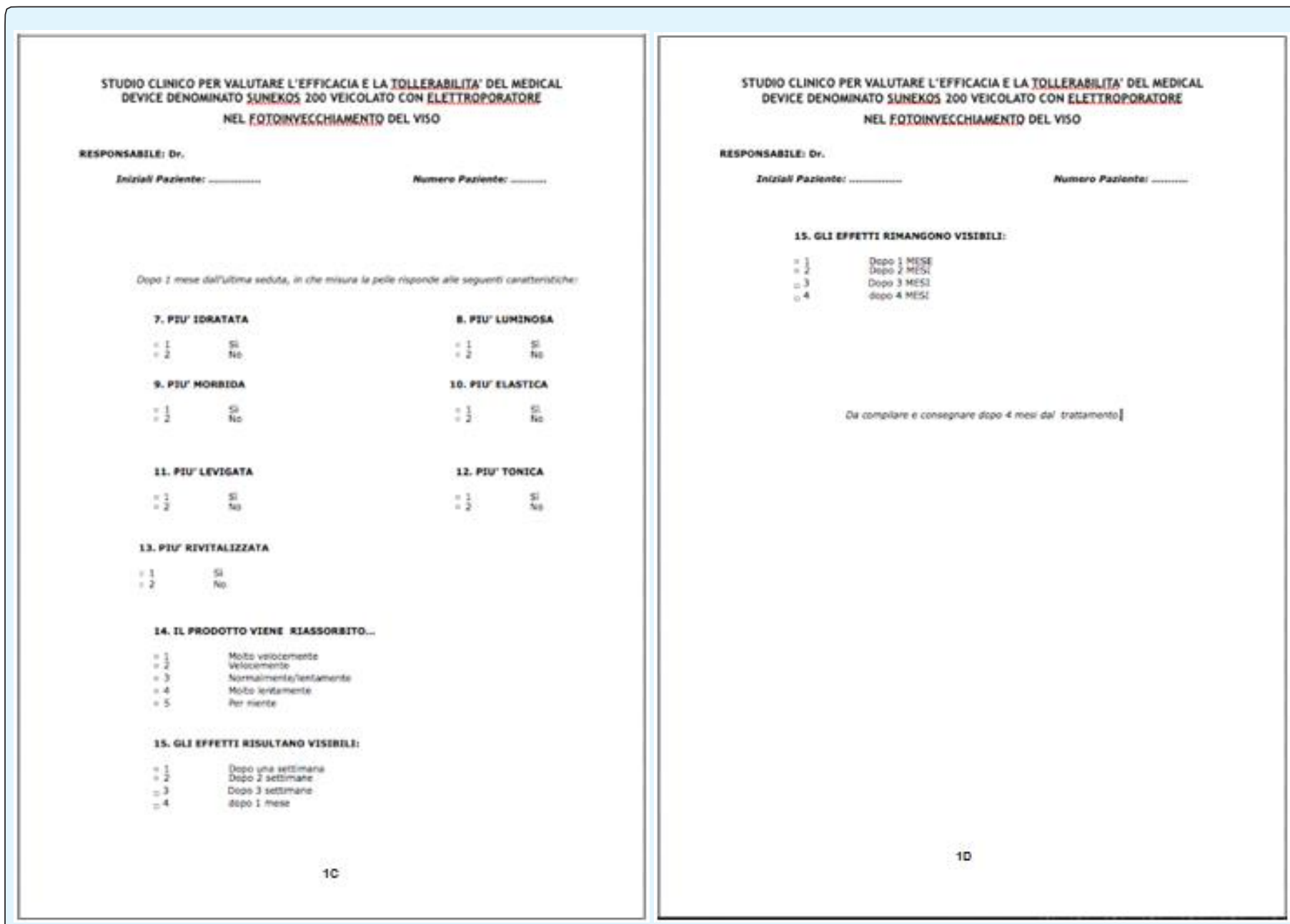

Figure 1A-1D: Study clinic to assess effectiveness and tolerability.

\section{Statistic Methodology}

A total of 30 patients registered in the study.

The statistical analyses reported below were divided up based on the type of instrument used to perform the measurements and the area treated (eyes and cheeks). A table containing some descriptive statistics is shown for each type of instrument used and, separately for eyes and cheeks; in details, for the continuous variables, the number of patients, average, median, range, standard error and confidence interval were calculated.

In addition to the table with the descriptive data, a table containing all the results of the statistical models performed was also added to assess any differences in the parameters measured at the basal time and in the discharging phase.

The statistical analyses designed to identify any differences in the values of the scores measured at the basal time and at the T1 times (end of the treatment) and T2 (one month after the end of the treatment), were performed using Linear mixed Models, in which the basal treatment (Basal, T1, T2) and the treated part (cheeks, eyes) were entered as fixed factors. The identity of the patient was used as a random factor for taking into account any differences solely ascribable to the subjectivity of the patient and remove these from the model, increasing power and accuracy. Four independent analyses were carried out, one for each measuring device. 


\section{Clinical Dermatology Open Access Journal}

In the diagram shown, the dots indicate the average value, while the bars represent the standard deviation. All the analyses were performed using the $\mathrm{R}$ ver software. 3.4.1

\section{Descriptive}

The tables below contain the descriptive statistics of the parameters; in detail. Average \pm standard error, median and range ( $\min -\max )$.

\begin{tabular}{|c|c|c|c|c|}
\hline & pH meter & Sebum meter & Dermis & Epithermometer \\
\hline \multirow{3}{*}{ basal } & $5.83 \pm 0.06$ & $19.33 \pm 3.23$ & $4.12 \pm 0.22$ & $28.05 \pm 0.18$ \\
\cline { 2 - 5 } & 5.75 & 16 & 4.1 & 28 \\
\cline { 2 - 5 } & $(5.3-6.9)$ & $(3-86)$ & $(1-6.8)$ & $(26-30)$ \\
\hline \multirow{3}{*}{ T1 } & $5.77 \pm 0.04$ & $23.37 \pm 3.49$ & $4.74 \pm 0.19$ & $28.09 \pm 0.18$ \\
\cline { 2 - 5 } & 5.7 & 17.5 & 5 & 28 \\
\hline \multirow{3}{*}{ T2 } & $(5.5-6.2)$ & $(4-88)$ & $(2-6.5)$ & $(26.5-30)$ \\
\cline { 2 - 5 } & $5.68 \pm 0.03$ & $28.37 \pm 3.59$ & $5.55 \pm 0.2$ & $28.33 \pm 0.16$ \\
\cline { 2 - 5 } & 5.7 & 21.5 & 5.55 & 28.5 \\
\hline
\end{tabular}

Table 1: Cheeks.

\begin{tabular}{|c|c|c|c|c|}
\hline & pH meter & Sebum meter & Dermis & Epithermometer \\
\hline \multirow{3}{*}{ basal } & $5.81 \pm 0.05$ & $18 \pm 2.48$ & $4 \pm 0.18$ & $27.42 \pm 0.26$ \\
\cline { 2 - 5 } & 5.8 & 12.5 & 4 & 27.75 \\
\cline { 2 - 5 } & $(5.5-7.1)$ & $(3-55)$ & $(2.1-6.5)$ & $(23.5-30)$ \\
\hline \multirow{3}{*}{ T1 } & $5.76 \pm 0.04$ & $21.1 \pm 2.4$ & $4.51 \pm 0.17$ & $27.47 \pm 0.24$ \\
\cline { 2 - 5 } & 5.8 & 17 & 4.6 & $(2.8-6.3)$ \\
\hline \multirow{3}{*}{ T2 } & $5.66 \pm 0.03$ & $(7-57)$ & $5.18 \pm 0.18$ & $27.72 \pm 0.21$ \\
\cline { 2 - 5 } & 5.6 & $24.3 \pm 2.4$ & 5.2 & 28 \\
\cline { 2 - 5 } & $(5.4-6)$ & 21 & $(2-6.9)$ & $(26-29.5)$ \\
\hline
\end{tabular}

Table 2: Eyes.

\section{Results}

In all the cases, a clear visual improvement was observed in the level of ageing of the skin, with a measurable improvement in the level of hydration of the skin and an improvement in the sebum levels. For the other measurable parameters, there were no significant variations. No signs of intolerance or hypersensitivity to the product were ever registered in any of the patients treated.

\section{Results of the Models}

pH Meter: The $\mathrm{pH}$ value fails to differ significantly between eyes and cheeks, but is significantly reduced with the treatment. The difference between basal and T1 is hardly significant in statistical terms, whereas the different between basal and $\mathrm{T} 2$ is highly significant (Table 3).

\begin{tabular}{|c|c|c|c|}
\hline Effect & F & Gdl & P \\
\hline Site (cheeks vs eyes) & 0,357 & 1.147 & 0.55 \\
\hline Treatment & 13,674 & 2.147 & $<\mathbf{0 . 0 0 1}$ \\
\hline
\end{tabular}

Table 3: pH Meter.

In detail

T1 vs basal, $\mathrm{t}=1.971$, $\mathrm{gdl}=147, \mathrm{P}=\mathbf{0 . 0 5 1}$

$\mathrm{T} 2$ vs basal, $\mathrm{t}=5.180, \mathrm{gdl}=147, \mathrm{P}=\mathbf{0 . 0 0 1}$

\section{Sebum meter}

The response does not change in relation to the area of application (eyes vs cheeks), but increases significantly with the treatment. As in the case of the pHmeter, the difference between basal and T1 is statistically negligible, 
Clinical Dermatology Open Access Journal

whereas the T2 basal difference is highly significant (Table 4).

\begin{tabular}{|c|c|c|c|}
\hline Effect & F & Gdl & P \\
\hline Site (cheeks vs eyes) & 2,735 & 1.147 & 0.1 \\
\hline Treatment & 8.2196 & 2.147 & $\mathbf{4 E - 0 4}$ \\
\hline
\end{tabular}

Table 4: Sebum meter.

In detail:

T1 vs basal, $\mathrm{t}=1.885$, gdl=147, $\mathrm{P}=0.061$

$\mathrm{T} 2$ vs basal, $\mathrm{t}=4.051, \mathrm{gdl}=147, \mathrm{P}=0.001$

\section{Dermis}

In this case, significant differences emerge, both for the areas of application and for the treatment. The response is considerably higher in the cheeks and develops significantly with the treatment. The measurement for T1 and $\mathrm{T} 2$ is significantly higher than the basal value (Table $5)$.
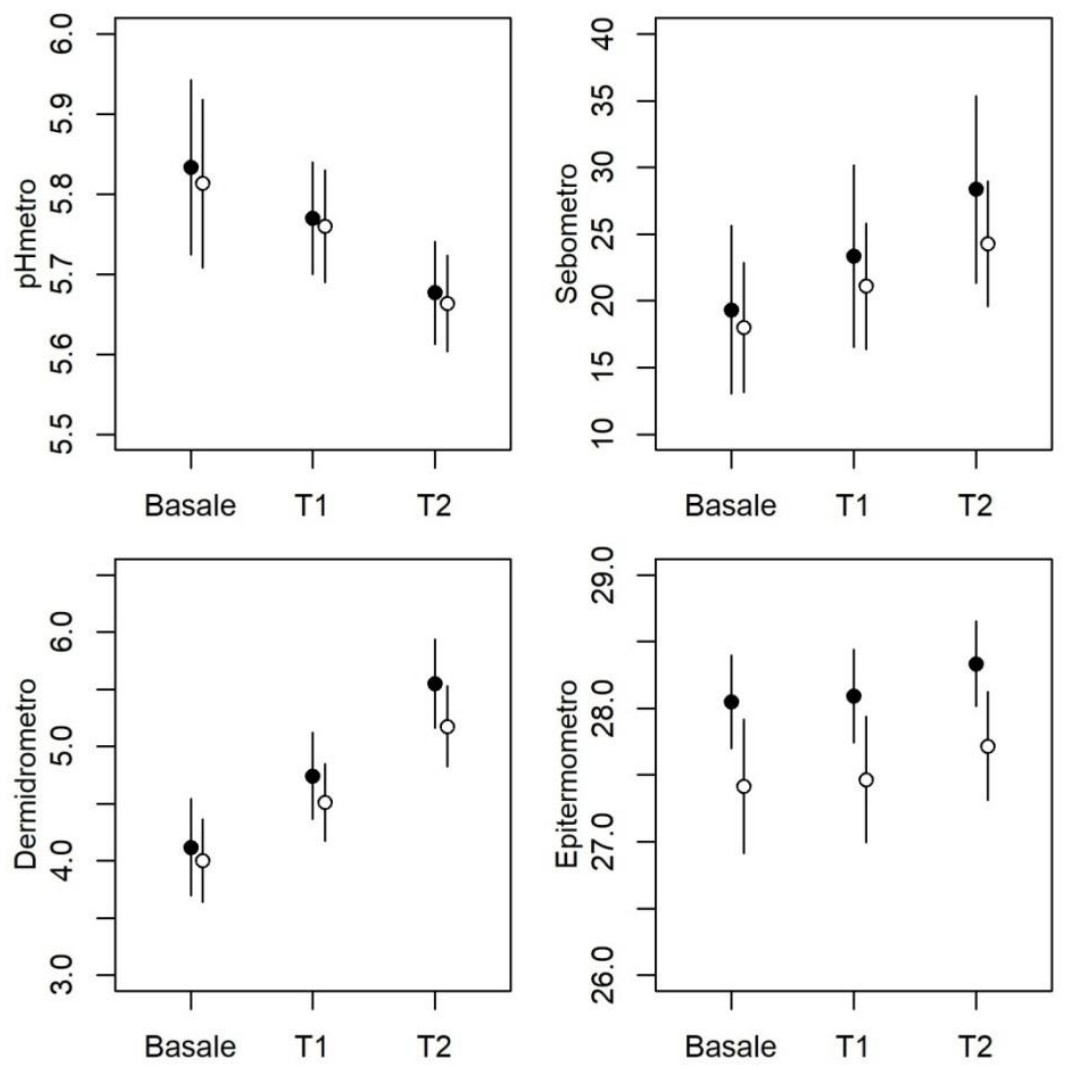

Figure 2: Instrumental assessment of the changes with regard to the basal observed on the skin at the end of the treatment and one month after the end of the treatment with reference to $\mathrm{pH}$, sebum, level of hydration and temperature. The symbols refer to cheeks (black dot) and eyes (white dot). 


\section{Discussion}

By performing objective and subjective clinical comparisons of the results obtained after by administration using the medical device being studied herein, and those obtained with the classical administration technique, intradermal treatment, in both cases an immediate "filler effect" was ascertained, with an epidermis that immediately appears tighter $[8,22]$.

The application technique performed with the electroporator is completely painless compared with the intradermal therapy technique and the - albeit raretypical side effects of intradermal therapy, such as small bruises and edemas, do not occur. In any case the most notable results appear in the space of about a month, when SUNEKOS 200, by stimulating the growth and multiplication of the collagen and elastin cells, produces a visible anti-ageing action. Wrinkles are visibly reduced and the skin looks tighter and fuller. In particularly dehydrated, deflated and saggy skins, hydration is gradually restored to the surface of the dermis $[9,18]$.

In patients who received treatment on their hands, the tissues were perfectly reintegrated, giving the skin on the hands a new fullness and plumpness and restoring tone.

\section{Conclusions}

The study performed on SUNEKOS 200, applied to the skin using electroporation, generated good results, improving the fullness and reducing the fine wrinkles on the surface, making the skin look fresh, bright and healthy again, as can be achieved using the intradermal therapy technique. However, it is not a filler treatment as it does not create volume, but in any case it improves both the level of hydration of the skin and the levels of sebum. Indeed, the results of the statistical models show that the $\mathrm{pH}$ value of the skin reduces but does not significantly change in the various areas of the face with the treatment, whereas the sebum values increase after the treatment all over the face.

The hydration of the skin improves in a statistically significant way, above all in the cheeks, as can be seen from the statistical tables attached. The results are less visible and long-lasting that the introduction of the device with intradermal therapy technique.

In the hands, the application with electroporation proved more effective, both due to the lack of side effects, typical of the infiltrative technique, such as swelling, pain and normal redness, and also due to the almost immediate results, as the hands immediately become softer and fuller.

This type of application is in any case preferable for patients with a phobia of needles, or with a particularly active life in the public eye, who cannot afford to show any blemishes on the facial skin (bruises, edemas, even if only light, etc.). Based on the objective results and those resulting from the clinical analyses, this typed of application method can be a valid alternative to the traditional intradermal therapy method.

\section{References}

1. (2011) Journal of Plastic Dermatology 7(1).

2. Farkas JP, Pessa JE, Hubbard B, Rohrich RJ (2013) The Science and Theory behind Facial Aging. Plast Reconstr Surg Glob Open 1(1): e8-e15.

3. Ascher B, Coleman S, Alster T, Bauer U, Burgess C, et al. (2006) Full scope of effect of facial lipoatrophy: a framework of disease understanding. Dermatol Surg 32(8): 1058-1069.

4. Han A, Chien AL, Kang S (2014) Photoaging. Dermatol Clin 32(3): 291-299.

5. Day DJ, Littler CM, Swift RW, Gottlieb S (2004) The wrinkle severity rating scale: a validation study. Am J Clin Dermatol 5(1): 49-52.

6. Glogau RG (1996) Aestethic and anatomic analysis of the aging skin. Semin Cutan Med Surg 15(3): 134-138.

7. Avantaggiato A, Bertuzzi G, Vitiello U, Iannucci G, Pasin M, et al. (2014) Role of antioxidants in dermal aging: an in vitro study by q-RT-PCR. Aesthetic Plast Surg 38(5): 1011-1016.

8. Boisnic S, Brancher MC (2006) Evaluation of the antiaging effect on human skin maintained in survival after injection of a mixture of hyaluronic acid and multivitamins with the meso injector u-med biopsy 225. J Med Esth and Chir Derm 33(132): 253-258.

9. Avantaggiato A, Girardi A, Palmieri A, Pascali M, Carinci F (2015) Comparison of Bio-Revitalizing Injective Products: A Study on Skin Fibroblast Cultures. Rejuvenation Res 18(3): 270-276. 
10. Hoffmann K (2009) Volumizing effects of a smooth, highly cohesive, viscous $20 \mathrm{mg} / \mathrm{mL}$ hyaluronic acid volumizing filler: prospective European study. BMC Dermatol 9: 9.

11. Sparavigna A, Forte R, Dioguardi FS (2007) Multicenter study for the evaluation of tolerance and efficacy of a new integrated aminoacid treatment on the aging face. J of Plastic Dermatol 3: 19-25.

12. Batheja $P$, Thakur R, Michniak B (2006) Transdermal iontophoresis.. Expert Opin Drug Deliv 3(1): 127-138.

13. Cornille P (2005) Mesolift, the soft. The official. Cosmetic Surgery, pp: 10.

14. Huteau Y (1999) The pharmacopoeia in mesotherapy. The documents of mfc.

15. Bernal, Thomas N (1999-2000) Mesotherapy applied to the treatment of facial wrinkles. The mesotherapy.

16. Juluri A, Peddikotla P, Repka MA, Murthy SN (2012) Transdermal iontophoretic delivery of propofol: A general anaesthetic in the form of its phosphate salt. J Pharm Sci 102(2): 500-507.
17. Dixit N, Bali V, Baboota S, Ahuja A, Ali J (2007) Iontophoresis - an approach for controlled drug delivery: a review. Curr Drug Deliv 4(1): 1-10.

18. Petit P (2005) Aesthetic mesotherapy face, neck and chest: a new youth through technology epidermal. 25Th national congress of aesthetic medicine and surgery dermatology. J Med Esth and Chir Derm 32(125): 45-63.

19. Vilain R, Godeau MJ Concerns of the aesthetic skin face. Masson Ch Vi Aging, pp: 166-175.

20. De Rigal J, Leveque JL (1985) In vivo measurement of the stratum corneum elasticity. Bioengineering and the skin 1: 13-23.

21. Grove GL, Grove MJ, Leyden JJ (1989) Optical Profilometry: an objective method for quantification of facial wrinkles. J Am Acad Dermatol 21(3): 631637.

22. De Servi B, Orlandini A, Caviola E, Meloni M (2016) Amino acids and hyaluronic acid mixtures differentially regulate extra cellular matrix genes in cultured human fibroblast (Ahead of Print). 